\title{
MEASUREMENT OF INSERTION LOSS VERSUS FIBER RECESSION IN FIBER OPTIC CONNECTORS
}

\author{
Eric Lindmark \\ PROMET International
}

Shoreview, $M N$

\section{Introduction}

A study was commissioned to analyze the effects of optical fiber recession/protrusion inside fiber optic connector and terminus ceramic ferrules. The purpose of fiber optic connectors is to provide a reusable, repeatable, reliable and low optical loss method of joining two separate pieces of optical fiber. In order to achieve these goals, various standards bodies have devised recommendations for the geometry of the polished endface of these connectors including the Spherical Fiber Height [1]. Fiber height is also referred to as fiber recession when the fiber is below the surface of the connector's ceramic ferrule and is typically measured with an optical interferometer specially designed for the purpose of measuring connector endface geometry. A typical acceptable range for fiber height is from -125 to +50 nanometers. The purpose of this study is to examine the optical performance when the Spherical Fiber Height is not within the recommended range.

\section{Theoretical Losses Due to Longitudinal Separation}

The main measure of optical performance between a joined pair of optical fiber connectors is insertion loss. It is the amount of light that is lost when transferring between the first fiber core to the second fiber core and is affected by how well the two fibers are aligned to each other. Connector loss is usually stated in decibels $(\mathrm{dB})$ as a ratio of two optical powers [2]. Connector loss increases rapidly when the cores are displaced laterally, and is not considered here. For a coherent source, the main loss mechanisms when the cores become axially displaced are separation loss from the numerical aperture of the fiber and FabryPerot interference effects from light bouncing back and forth between the connector endfaces when the fibers are no longer touching $[3,4,5]$.

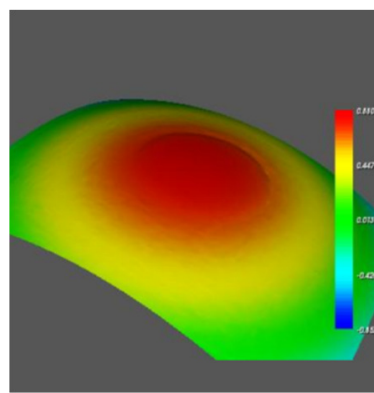

$+21 \mathrm{~nm}$

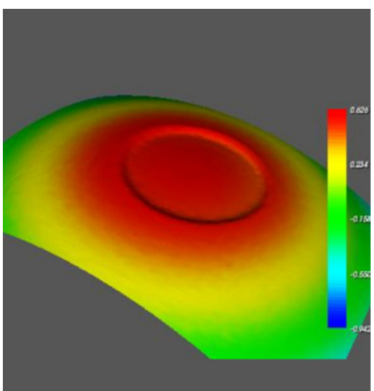

$-123 \mathrm{~nm}$

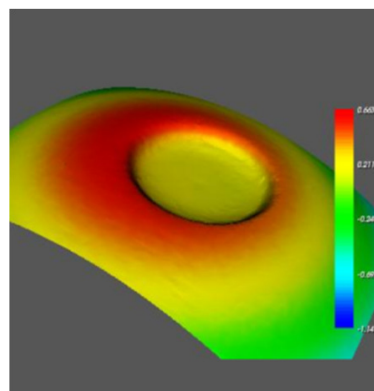

$-509 \mathrm{~nm}$

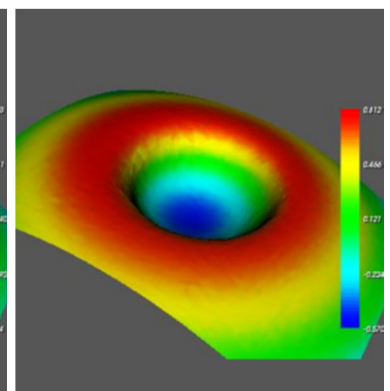

$-1202 \mathrm{~nm}$

Figure 1. Example 3D Endface Images with Different Spherical Fiber Heights from Polishing

\section{Experimental Results}

SC/PC style connectors were constructed, and using a proprietary polishing method that attacked the fiber preferentially (see Figure 1.), the connector endface was polished in steps. After each polishing step, the connector endface's fiber height was measured on a $\mathrm{FiBO}^{\circledR}$ interferometer and insertion loss was 
measured while being coupled to a reference connector with a similar radius of curvature and near zero fiber height.

For two connectors measured using this method using two different wavelength lasers, the resulting data is shown in Figure 2:
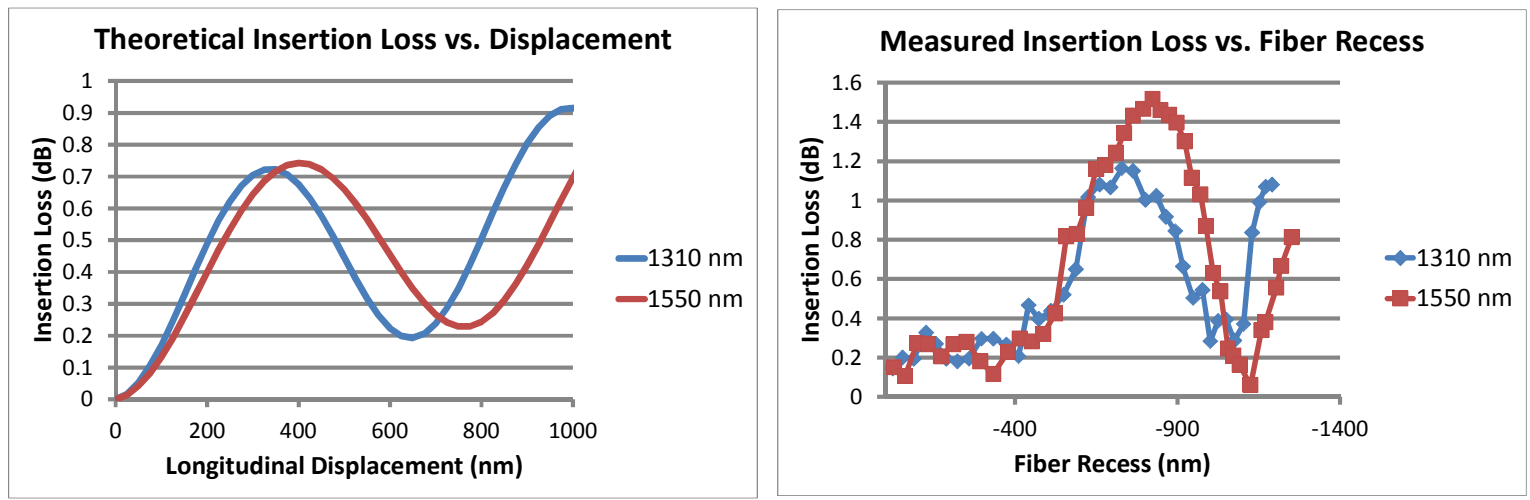

Figure 2. Theoretical \& Measured Insertion Losses versus Fiber Recess

\section{Conclusion}

Physical endface separation does not occur until after about 400 nanometers. The point of separation can vary with the specific geometries of both mating fibers and the amount of compression force at the contact point. When the endfaces are no longer in physical contact, they demonstrate Fabry-Perot interference. This etalon effect causes the insertion loss to initially rise much more rapidly than would be expected with strictly NA calculations, but then can go almost back to zero after the initial peak in insertion loss. Extra loss seen in the measured data is most likely due to imperfections in the connectors, secondary signal loss effects and measurement uncertainty.

Using lasers of different wavelengths shows an effect upon the etalon; as the wavelength decreases, the peaks of the etalon effect get closer together because their spacing is directly related to the wavelength of the laser used.

\section{References}

[1] Telecommunications Industry Association, "TIA Standard TIA-455-218, FOTP-218, Measurement of Endface Geometry of Single Fiber Optical Connectors," Arlington, Virginia, 2002.

[2] Dennis Derickson, Fiber Optic Test and Measurement, Chapter 9, Prentice Hall PTR, Upper Saddle River, NJ, 1998.

[3] F.L. Thiel and R.M. Hawk, “Optical waveguide cable connection,” Applied Optics, vol. 15, no. 11, pp. 27852791, 1976.

[4] D.L. Bisbee, "Measurements of Loss Due to Offsets and End Separations of Optical Fibers," The Bell System Technical Journal, vol. 50, no. 10, pp. 3159-3168, 1971.

[5] Eugene Hecht, Optics $2^{\text {nd }}$ ed., Chapter 9, Addison-Wesley Publishing Company, Reading, Massachusetts, 1987.

\section{Acknowledgement}

This work was performed under a NAVAIR Small Business Innovative Research contract. 